\title{
Outcome After PSMA PET/CT-Based Salvage Radiotherapy in Patients with Biochemical Recurrence After Radical Prostatectomy: A 2-Institution Retrospective Analysis
}

\author{
Nina-Sophie Schmidt-Hegemann ${ }^{1}$, Christian Stief ${ }^{2}$, Tak-Hyun Kim ${ }^{3}$, Chukwuka Eze ${ }^{1}$, Simon Kirste ${ }^{3}$, Iosif Strouthos ${ }^{3}$, \\ Minglun Li ${ }^{1}$, Wolfgang Schultze-Seemann ${ }^{4}$, Harun Ilhan ${ }^{5}$, Wolfgang Peter Fendler ${ }^{6}$, Peter Bartenstein ${ }^{5}$, Anca-Ligia Grosu ${ }^{3,7}$, \\ Ute Ganswindt ${ }^{8}$, Claus Belka ${ }^{1,9}$, Philipp T. Meyer ${ }^{10}$, and Constantinos Zamboglou ${ }^{3,7,11}$ \\ ${ }^{I}$ Department of Radiation Oncology, University Hospital, LMU Munich, Munich, Germany; ${ }^{2}$ Department of Urology, University \\ Hospital, LMU Munich, Munich, Germany; ${ }^{3}$ Department of Radiation Oncology, Faculty of Medicine, Medical Center, University \\ of Freiburg, Freiburg, Germany; ${ }^{4}$ Department of Urology, Faculty of Medicine, Medical Center, University of Freiburg, Freiburg, \\ Germany; ${ }^{5}$ Department of Nuclear Medicine, University Hospital, LMU Munich, Munich, Germany; ${ }^{6}$ Department of Nuclear \\ Medicine, University Hospital Essen, Essen, Germany; ${ }^{7}$ German Cancer Consortium (DKTK), Partner Site Freiburg, Germany; \\ ${ }^{8}$ Department of Therapeutic Radiology and Oncology, Innsbruck Medical University, Innsbruck, Austria; ${ }^{9}$ German Cancer \\ Consortium (DKTK), Partner Site Munich, Germany; ${ }^{10}$ Department of Nuclear Medicine, Faculty of Medicine, Medical Center, \\ University of Freiburg, Freiburg, Germany; and ${ }^{11}$ Berta-Ottenstein-Programme, Faculty of Medicine, Medical Center, University \\ of Freiburg, Germany
}

Prostate-specific membrane antigen (PSMA) PET/CT detects prostate cancer recurrence at low levels of prostate-specific antigen (PSA). Radiotherapy with dose escalation to the former prostate bed has been associated with improved biochemical recurrence-free survival (BRFS). Thus, we hypothesized that PSMA PET/CT-guided salvage radiotherapy leads to improved BRFS. Methods: In total, 204 consecutive patients were referred for salvage radiotherapy after radical prostatectomy. PSMA PET/CT scans were performed, and patients with PSA persistence (109 patients) or evidence of distant metastases (5 patients) were excluded from this analysis. Thus, the following analysis is based on a total of 90 patients who underwent PSMA PET/CT before radiotherapy due to biochemical recurrence and received salvage radiotherapy. In cases of PET-positive findings, antiandrogen therapy was commenced before initiation of radiotherapy. BRFS (PSA $\leq 0.2 \mathrm{ng} / \mathrm{mL}$ ) was defined as the study endpoint. Results: PET-positive lesions were detected in 42 of 90 (47\%) patients, 24 of $42(27 \%)$ being fossa recurrence only, 12 of $42(13 \%)$ pelvic lymph node only, and 6 of $42(7 \%)$ both fossa and pelvic lymph node. The median PSA before radiotherapy was $0.44 \mathrm{ng} / \mathrm{mL}$ (range, $0.11-6.24 \mathrm{ng} / \mathrm{mL}$ ). Cumulatively, a total dose of $70.0 \mathrm{~Gy}$ (range, 67.2-72 Gy) was delivered to local macroscopic tumor, 66 Gy (range, 59.4-70.2 Gy) to the prostatic fossa, $60.8 \mathrm{~Gy}$ (range, 54-66 Gy) to PETpositive lymph nodes, and $50.4 \mathrm{~Gy}$ (range, 45-50.4 Gy) to the lymphatic pathways. After a median follow-up of 23 mo, BRFS was $78 \%$. Antiandrogen therapy was ongoing in 4 patients at the last follow-up. No significant difference in BRFS between PET-positive patients (74\%) and PET-negative patients $(82 \% ; P>0.05)$ was observed at the last follow-up. Two patients had late genitourinary toxicity, grade 3 , and no patient had gastrointestinal toxicity of grade 3 or higher (National Cancer Institute common terminology criteria for adverse events, version 4.03). Conclusion: PSMA PET/CT-guided salvage radiotherapy is an effective and safe local treatment option. No difference in BRFS

Received Apr. 8, 2018; revision accepted Jun. 23, 2018.

For correspondence or reprints contact: Nina-Sophie Schmidt-Hegemann, Department of Radiation Oncology, University Hospital, LMU Munich, Marchioninistrasse 15, 81377 Munich, Germany.

E-mail: nina-sophie.hegemann@med.uni-muenchen.de

Published online Jul. 12, 2018.

COPYRIGHT (C) 2019 by the Society of Nuclear Medicine and Molecular Imaging. between PET-positive and PET-negative patients was observed, indicating effective targeting of PET-positive lesions. PSMA PET/ CT when readily available should be offered to patients with PSA recurrence for treatment individualization.

Key Words: prostate cancer; PSMA PET/CT; biochemical recurrence; radiotherapy

J Nucl Med 2019; 60:227-233

DOI: 10.2967/jnumed.118.212563

A lmost $30 \%$ of prostate cancer (PCa) patients have a biochemical relapse after radical prostatectomy within the first $5 \mathrm{y}$ (1). In this case, early salvage radiation therapy (sRT) of the former prostate bed with or without androgen deprivation therapy (ADT) (2) is recommended. A dose-response relationship for recurrent $\mathrm{PCa}$ was described in several studies, suggesting that radiotherapy doses of more than 70 Gy should be applied to the prostatic fossa (3). However, the SAKK 09/10 study showed that dose escalation had a negative impact on patients' quality of life regarding urinary symptoms after sRT (4). Consequently, an accurate detection of the individual PCa distribution is urgently required to select suitable patients for sRT and individualize radiotherapy dose escalation within the pelvis.

In the last few years, prostate-specific membrane antigen (PSMA) PET/CT has rapidly evolved to be the gold standard in staging patients with biochemical relapse: Eiber et al. reported high detection rates for PSMA PET/CT in patients with recurrent $\mathrm{PCa}$, especially for patients with prostate-specific antigen (PSA) levels of 0.5 to $<1 \mathrm{ng} / \mathrm{mL}(73 \%)$ and 0.2 to $<0.5 \mathrm{ng} / \mathrm{mL}$ (58\%) (5). Jilg et al. proved that PSMA PET has a good sensitivity and specificity in lymph node detection for patients with biochemical relapse (6). Keeping in mind that early salvage radiotherapy should start at PSA levels of less than $0.5 \mathrm{ng} / \mathrm{mL}$, the latter observations already indicate that PSMA PET/CT can influence the decision-making process before initiation of sRT. Indeed, several studies showed that PSMA 
PET/CT leads to a significant change in management of patients with biochemical relapse after surgery (7-10).

Several studies reported their outcomes after non-PSMA PET/ CT-guided sRT (11-13) and reported biochemical control in less than $70 \%$ of their patients after 2 y. Zschaeck et al. performed PSMA PET-guided sRT in 22 high-risk PCa patients with biochemical relapse and showed that a prolonged PSA response was achieved in hormone-naïve patients (14). Emmet et al. performed PSMA PETguided sRT in 146 patients. After a median follow-up of $10.5 \mathrm{mo}$, an overall treatment response of $75 \%$ was reported (15).

The purpose of this German 2-institution retrospective analysis was to assess PSA response after PSMA PET/CT-based individualization of sRT: standard radiotherapy in cases of biochemical relapse constitutes irradiation of the prostatic fossa only. Further individualization in the sense of dose escalation to macroscopic local recurrences in the prostatic fossa and irradiation of pelvic lymphatic pathways with escalated dose to PET-positive lymph nodes and initiation of ADT was based on the findings in PSMA PET/CT. Further, biochemical recurrence-free survival (BRFS) of this treatment-intensified cohort was compared with historical data before the advent of PSMA PET/CT.

\section{MATERIALS AND METHODS}

\section{Patient Population}

From February 2014 onward, 204 consecutive patients were referred for salvage radiotherapy after radical prostatectomy due to persistent or rising PSA at the departments of radiation oncology of the university medical centers of Munich and Freiburg. PSMA PET/ CT scans were performed, and patients with PSA persistence (109 patients) were excluded from this analysis because their data regard tumor stage at the time of radical prostatectomy and are not comparable with the data of patients who have a PSA decrease after prostatectomy (16). Additionally, patients with PSA recurrence fare much better than patients with PSA persistence pertaining to BRFS, distant metastasis-free survival, and overall survival $(16,17)$. Five patients with a first PSA recurrence after radical prostatectomy showed evidence of distant metastases on PSMA PET/CT (1/5 [20\%] had nonregional lymph node metastases and 4/5 [80\%] had bone metastases) and were also excluded. Thus, the following analysis is based on a cohort of 90 patients who underwent PSMA PET/CT before radiotherapy due to biochemical relapse (Table 1; Supplemental Fig. 1 [supplemental materials are available at http://jnm.snmjournals.org]). In these 90 patients, PSA recurrence was newly diagnosed without any salvage treatment beforehand. This retrospective analysis was performed in compliance with the principles of the Declaration of Helsinki and its subsequent amendments (18) and was approved by the local Ethics Committee of the respective medical faculties (University of Freiburg approval 519/17; University of Munich approval 17-765). The requirement to obtain informed consent was waived.

\section{Treatment Application and Follow-up}

Treatment management after PSMA PET was documented for each patient. Follow-up time was defined as the interval in months between radiotherapy and the last recorded PSA. Follow-up examination was first performed 6 wk to 3 mo after sRT and then every 6-12 mo. Patients were regarded free of ADT influence after a minimum of 2 mo after the last application of ADT.

\section{PSMA Ligand and PET/CT Imaging}

PSMA-HBED-CC was radiolabeled according to good clinical practice at both medical centers as described previously $(19,20)$. At
TABLE 1

Patient Characteristics

\begin{tabular}{|c|c|}
\hline Characteristic & Data \\
\hline Total patients $(n)$ & 90 \\
\hline Median age $(\mathrm{y})$ & 73 \\
\hline \multicolumn{2}{|l|}{ Tumor stage $(n)$} \\
\hline $\mathrm{pT} 2 \mathrm{a} / \mathrm{b}$ & $10(11 \%)$ \\
\hline pT2c & $44(49 \%)$ \\
\hline рT3a & $23(26 \%)$ \\
\hline pT3b & $11(12 \%)$ \\
\hline pT4 & $2(2 \%)$ \\
\hline \multicolumn{2}{|l|}{ Nodal stage $(n)$} \\
\hline pNO & $66(73 \%)$ \\
\hline $\mathrm{pN} 1$ & $11(12 \%)$ \\
\hline $\mathrm{pNx} / \mathrm{cNO}$ & $13(15 \%)$ \\
\hline Positive surgical margins $(n)$ & $28(31 \%)$ \\
\hline \multicolumn{2}{|l|}{ Gleason score $(n)$} \\
\hline 6 & $8(9 \%)$ \\
\hline $7 a$ & $25(27 \%)$ \\
\hline $7 b$ & $33(37 \%)$ \\
\hline 8 & $15(17 \%)$ \\
\hline 9 & $9(10 \%)$ \\
\hline $\begin{array}{l}\text { Median PSA at radical } \\
\text { prostatectomy }(\mathrm{ng} / \mathrm{mL})\end{array}$ & 8.55 (range, 2.37-48.0) \\
\hline Postoperative PSA (ng/mL) & 0.03 (range, $0.0-0.19$ ) \\
\hline $\begin{array}{l}\text { Mean months since radical } \\
\text { prostatectomy until PSA } \\
\text { recurrence }\end{array}$ & 62 (range, 8-199) \\
\hline $\begin{array}{l}\text { Median PSA at PSMA PET/CT } \\
(\mathrm{ng} / \mathrm{mL})\end{array}$ & $0.43(0.10-6.24)$ \\
\hline $\begin{array}{l}\text { Median PSA at PSMA PET/CT } \\
\text { in } 42 \text { PET-positive patients } \\
\text { (ng/mL) }\end{array}$ & $0.78(0.23-6.24)$ \\
\hline $\begin{array}{l}\text { Median PSA at PSMA PET/CT } \\
\text { in } 48 \text { PET-negative patients } \\
\text { (ng/mL) }\end{array}$ & $0.30(0.1-3.24)$ \\
\hline \multicolumn{2}{|l|}{ PSMA PET/CT result $(n)$} \\
\hline No recurrence & $48(53 \%)$ \\
\hline Fossa recurrence only & $24(27 \%)$ \\
\hline Lymph node recurrence only & $12(13 \%)$ \\
\hline $\begin{array}{l}\text { Fossa and lymph node } \\
\text { recurrence }\end{array}$ & $6(7 \%)$ \\
\hline
\end{tabular}

the University Medical Centre Freiburg, scans were performed with either a 64-slice Gemini TF PET/CT scanner or a 16-slice Gemini TF Big Bore PET/CT scanner (Philips Healthcare). Both scanners were cross-calibrated to ensure the comparability of the quantitative measurements. At the University Medical Center Munich, scans were performed with either a Siemens Biograph 64 or GE Healthcare Discovery 690. Phantom studies based on the National Electrical Manufacturers Association NU2-2001 standard were conducted in Munich to allow for pooling of the different scanner results. At the time of the PET scan, contrast-enhanced diagnostic CT (120 kVp, 100-400 mAs, dose modulation) or low-dose CT $(120 \mathrm{kVp}, 25 \mathrm{mAs})$ for attenuation correction (depending on previous CT scans and contraindications) 
was performed. PET scans were acquired at both institutions 60 min after intravenous administration of ${ }^{68} \mathrm{Ga}$-PSMA (mean, $205 \mathrm{MBq}$ ). Barring any contraindications, patients received 20 $\mathrm{mg}$ of furosemide at the time of tracer injection to avoid bladder activity.

\section{Image Interpretation}

PET/CT images were interpreted by 1 nuclear medicine physician and 1 radiologist or by 2 nuclear medicine physicians in the sense of a clinical report-based analysis. At least 1 of the readers had more than $5 \mathrm{y}$ of PET/CT experience. The location of each lesion was determined by CT. PET-positive lesions were visually identified by ${ }^{68} \mathrm{Ga}$-PSMA uptake above the background level and not associated with physiologic uptake (21).

\section{Radiotherapy}

All patients received intensity-modulated radiotherapy or volumetric modulated arc therapy and image-guided radiotherapy (2-5 times per week). Radiotherapy dose regimens were normofractionated or slightly hypofractionated, with a boost to the PET-positive lesions applied simultaneously or sequentially. Planning CT was done with the patient supine. Patients were advised to have a full bladder and empty rectum. The target was delineated according to the Radiation Therapy Oncology Group atlas for salvage PCa (22) and for pelvic lymph node delineation (23). The region of the former prostate gland is defined superiorly as $5 \mathrm{~mm}$ above the inferior border of the vas deferens remnant, inferiorly as above the top of the penile bulb, anteriorly by the pubic symphysis, posteriorly by the anterior rectum and laterally by the medial edge of the obturator internus muscle. The planning target volume was derived by expanding the clinical target volume by a $5-$ to $7-\mathrm{mm}$ margin in all directions.

\section{Statistical Analysis}

BRFS (PSA $\leq 0.2 \mathrm{ng} / \mathrm{mL}$ ) was defined as the study endpoint. For statistical analysis, SPSS Statistics, version 24 (IBM), was used. Time-to-event data were calculated using the Kaplan-Meier method. Differences between subgroups were compared using the $\log$ rank test, with a $P$ value of less than 0.05 considered statistically significant. Uni- and multivariate Cox-regression analyses were used to identify predictors for BRFS after PSMA PET/CTbased sRT.

\section{RESULTS}

\section{PSMA PET Results}

Around half the patients $(48 / 90 ; 53 \%)$ were PET-negative. If PET-positive, patients primarily had evidence of local recurrence $(24 / 42 ; 27 \%)$, followed by pelvic lymph node recurrence $(12 / 42$; $13 \%)$ and combined local and pelvic lymph node recurrence $(6 / 42$; $7 \%$ ). The median PSA at the time of PET/CT for all patients was $0.43 \mathrm{ng} / \mathrm{mL}$ (range, $0.10-6.24 \mathrm{ng} / \mathrm{mL}$ ). A positive PET scan was significantly $(P<0.01$, Fisher exact test) associated with higher PSA levels: $70 \%$ of these patients had a PSA level of more than $0.5 \mathrm{ng} / \mathrm{mL}$, whereas $81 \%$ of patients with a negative scan had a PSA level of $0.5 \mathrm{ng} / \mathrm{mL}$ or less (Table 1).

\section{Management of PET-Positive Lesions}

ADT was recommended to patients with evidence of PETpositive lesions for $2 \mathrm{y}$ (2). Patients with no PET-positive findings on PET/CT were normally treated with sRT alone. Consequently, 25 of $42(60 \%)$ PET-positive patients were started on ADT before initiation of sRT, 23 of 25 patients $(92 \%)$ chose to discontinue ADT after a median of 5 mo (range, 2-23 mo), and 17 of 42 (40\%) patients refused ADT altogether. One patient with no PET-positive findings received ADT concomitantly with radiotherapy and thereafter, because of a Gleason score of 9 and pathologic stage T3b. In all but 1 patient, the prostatic fossa was irradiated, with a median total dose of 66 Gy (range, 59.4-70.2 Gy) in 2.0-Gy fractions. In cases of PET-positive pelvic lymph nodes, pelvic lymphatic pathways were treated with a normofractionated overall dose of 45$50.4 \mathrm{~Gy}$. At the discretion of the treating physician, the boost to the PET-positive lymph nodes was delivered either simultaneously (15/18 [83\%]; 54-61.6 Gy in 1.9- to 2.4-Gy fractions) or sequentially (3/18 [17\%]; 9-12.6 Gy in 1.8-Gy fractions). Likewise, a simultaneous integrated boost (13/30 [43\%]; 70.2 Gy in 2.1- to 2.4-Gy fractions) or sequential boost (17/30 [57\%]; 3.8-25.2 Gy in 1.8-Gy fractions) was applied in cases of local recurrence on PET/CT. The aforementioned patient whose prostatic fossa was not irradiated had a low risk of local recurrence and a single internal iliac lymph node metastasis on PET/CT. He received irradiation of the pelvic lymphatic pathways only, with a simultaneous integrated

TABLE 2

Treatment Characteristics

\begin{tabular}{lccc}
\hline \multicolumn{1}{c}{ Characteristic } & All patients $(n=90)$ & PET-positive $(n=42)$ & PET-negative $(n=48)$ \\
\hline ADT with stop before last follow-up/duration $(\mathrm{mo})$ & $23 / 5(2-23)$ & $23 / 5(2-23)$ & - \\
\hline Ongoing ADT at last follow-up $(n)$ & 3 & 17 & 47 \\
No ADT $(n)$ & 64 & $0.68(0.3-6.24)$ & $0.34(0.11-3.24)$ \\
Median PSA before radiation therapy $(\mathrm{ng} / \mathrm{mL})$ & $0.44(0.11-6.24)$ & & \\
Radiation therapy $(\mathrm{Gy})$ & & & \\
\hline Former prostate & $66(59.4-70.2)$ & & \\
Lymphatic pathways & $50.4(45-50.4)$ & & \\
PET-positive local recurrence & $70.0(67.2-72)$ & & \\
PET-positive lymph node & $60.76(54-66)$ & & \\
\hline
\end{tabular}

${ }^{*}$ Radiation therapy technique for all patients was volume-modulated arc therapy/intensity-modulated radiotherapy and image-guided radiotherapy.

Data in parentheses are ranges. 
boost to the PET-positive lymph node metastasis. Treatment characteristics are listed in Table 2.

\section{Patients' Outcome}

The median follow-up was 23 mo (range, 1-47 mo). The median post-sRT PSA nadir was $0.07 \mathrm{ng} / \mathrm{mL}$ (range, $<0.03-1.18 \mathrm{ng} / \mathrm{mL}$ ), with 69 of 90 patients $(77 \%)$ presenting with a PSA of $0.1 \mathrm{ng} / \mathrm{mL}$ or less and 73 of $90(81 \%)$ with a PSA of $0.2 \mathrm{ng} / \mathrm{mL}$ or less. The median and mean times between end of radiotherapy and PSA nadir were 3 and 4.89 mo (range, 1-21 mo), respectively. At the time of PSA nadir measurement, 13 of 90 patients $(14 \%)$ had ongoing ADT and 6 of 90 patients (7\%) were still under the influence of ADT. At the last follow-up, the median PSA was $0.07 \mathrm{ng} / \mathrm{mL}$ (range, $<0.03-4.92 \mathrm{ng} / \mathrm{mL}$ ), with $78 \%$ of all patients having a PSA of $0.2 \mathrm{ng} / \mathrm{mL}$ or less (Fig. 1). Twenty-three of 26 patients $(88 \%)$ with concomitant ADT discontinued ADT after SRT: the median time between the last application of ADT and the last PSA measurement was 19 mo (range 2-36 mo). Sixty-nine of 87 patients $(80 \%)$ without ADT at the last follow-up had a PSA of $0.2 \mathrm{ng} / \mathrm{mL}$ or less at the last contact. No significant difference between patients with and without PET-positive findings before sRT regarding BRFS was observed at the last follow-up (78\% vs. $82 \%, P=0.392$; Fig. 2). Additionally, in neither PET-positive nor PET-negative patients did the use of ADT influence BRFS at the last follow-up $(P=0.410$; Fig. 3$)$. The patients' outcome after sRT is summarized in Table 3.

\section{Factors Predicting PSA Response at Last Follow-up}

Uni- and multivariate analysis was conducted to assess whether there was an association between BRFS and tumor- or treatmentspecific variables (Supplemental Table 1) and PSMA PET/CT imaging results. A postradiotherapy PSA nadir of $0.1 \mathrm{ng} / \mathrm{mL}$ or less was an independent factor for BRFS irrespective of ongoing ADT. All other factors had no significant impact on BRFS.

\section{Toxicity}

Acute genitourinary and gastrointestinal toxicity of grade 2, according to the Common Terminology Criteria for Adverse Events, version 4.03 , was observed in 12 of 90 patients $(13 \%)$ and 14 of 90 patients (16\%), respectively. Late genitourinary and

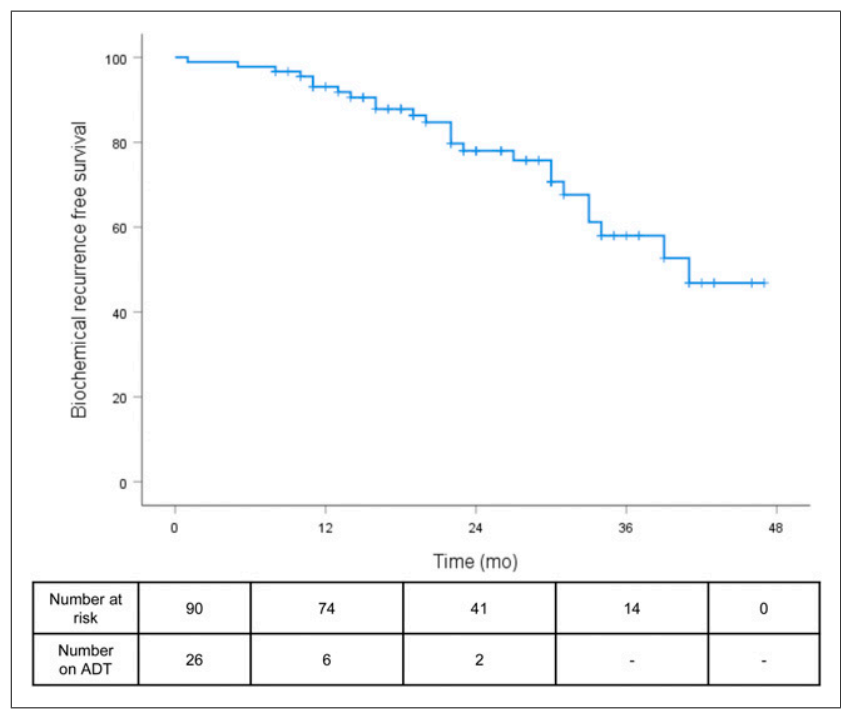

FIGURE 1. BRFS $(\leq 0.2 \mathrm{ng} / \mathrm{mL})$ in all patients at last follow-up.

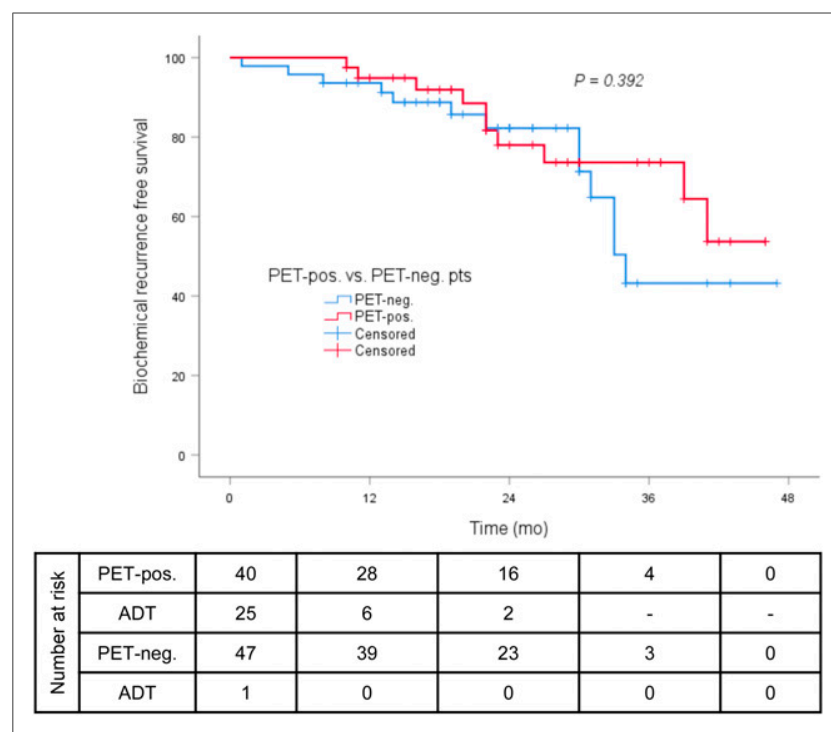

FIGURE 2. BRFS $(\leq 0.2 \mathrm{ng} / \mathrm{mL})$ in all patients without antiandrogen therapy at last follow-up (PET-positive vs. PET-negative).

gastrointestinal toxicity, grade 2, was seen in 12 of 90 patients $(13 \%)$ and 3 of 90 patients $(3 \%)$, respectively. There were 2 patients with late genitourinary toxicity, grade 3-one with radiation or noninfectious cystitis and the other with urinary incontinence. No patients had grade 4 or 5 toxicity (Supplemental Table 2).

\section{DISCUSSION}

The main aim of this study was to assess whether the excellent results of PSMA PET/CT in the detection of recurrent PCa after radical prostatectomy translate into improved BRFS for patients treated with PSMA PET-guided sRT.

As already extensively studied (10,24,25), PSMA PET/CT leads to remarkable treatment adaptation. In 42 of 90 patients $(47 \%)$, PET-positive recurrent disease was detected, hence triggering dose escalation, enlargement of radiotherapy volumes, and initiation of ADT.

For a median follow-up of $23 \mathrm{mo}$, our analysis showed that there is a high rate and long duration of treatment response to irradiation based on pretreatment PSMA PET/CT, with an overall low toxicity profile. At the last follow-up, $78 \%$ of patients had a PSA of $0.2 \mathrm{ng} / \mathrm{mL}$ or less. When the analysis was restricted to patients without ADT at the last follow-up, the benefit of PSMA PET/CT-based sRT became even more evident: $80 \%$ with a PSA of $0.2 \mathrm{ng} / \mathrm{mL}$ or less.

Numerous studies have reported on outcome after non-PSMA PET/CT-based sRT, with a varying proportion of patients having accompanying ADT or pelvic lymphatic pathway irradiation and with patients having differing pre-sRT PSA levels (Table 4) $(2,12,13,26-29)$. In comparison to studies in the pre-PSMA PET/ CT era, our results on BRFS after a median of 23 mo were favorable. Seemingly, the individualized intensification of treatment, exclusion of patients with distant metastases, and early initiation of salvage radiotherapy at a median PSA of $0.44 \mathrm{ng} / \mathrm{mL}$ explain these findings. Furthermore, this result mirrors a recent recommendation by the American Society for Radiation Oncology to start with sRT as early as possible (30). Our observations are largely in accordance with the limited number of analyses on outcome after PSMA PET/ CT-based sRT in recurrent PCa with mostly shorter follow-up 


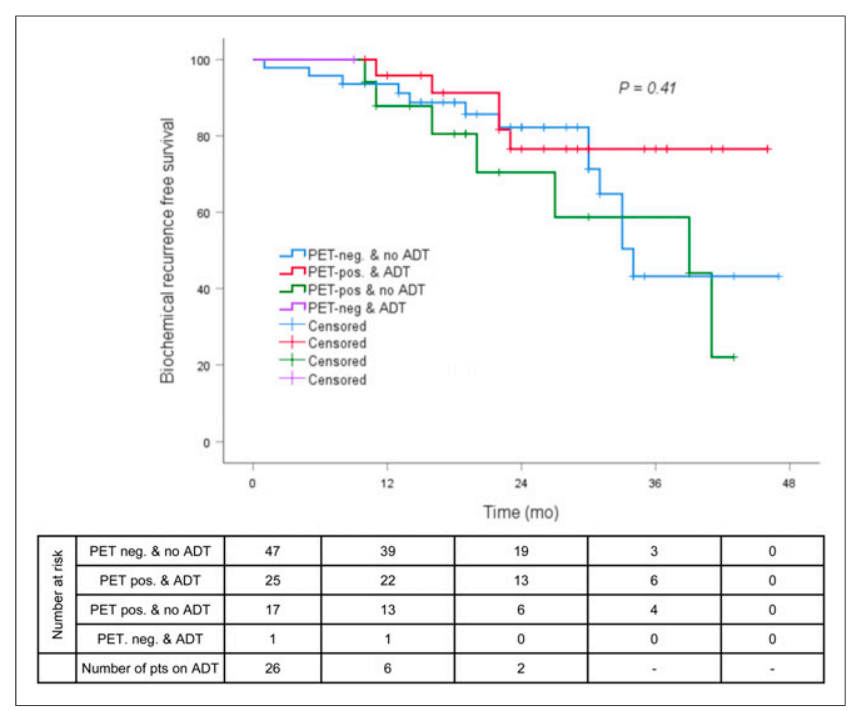

FIGURE 3. BRFS $(\leq 0.2 \mathrm{ng} / \mathrm{mL})$ in PET-positive vs. PET-negative patients with or without concomitant ADT.

$(14,15,31)$. Zschaeck et al. (14) reported on PSMA-based sRT in 20 patients with recurrent high-risk PCa. After a median follow-up of $29 \mathrm{mo}$, the median PSA was $0.15 \mathrm{ng} / \mathrm{mL}$. This corresponds well to the median PSA of $0.07 \mathrm{ng} / \mathrm{mL}$ after 23 mo in our cohort. Besides, Emmett et al. (15) reported on treatment outcome after PSMA PETbased radiotherapy with a shorter median follow-up of 10.5 mo and observed an overall treatment response of $72 \%$ (71/99 patients). This response is lower than that in our analysis, most likely because of their inclusion of patients with metastatic disease beyond nodal involvement within the pelvis and possibly of patients with persistent postprostatectomy PSA, although this is not clearly stated. The authors reported that nodal involvement in PSMA PET had a negative influence on BRFS, compared with patients with no PET findings or PET-positive lesions restricted to the fossa. In our analysis, neither PET-positive lesions in general nor PET-positive lymph nodes had an impact on BRFS. When first observed, this result is surprising, as one would assume that patients with visible recurrent disease would have a worse BRFS. First, it is not clearly stated whether the patients analyzed by Emmett et al. received irradiation of PET-positive lymph nodes only or of the surrounding lymphatic pathways as well, as was the case with our patients. Second, contrary to Emmett et al., we did not exclude patients who received ADT concomitantly with radiotherapy. Thus, treatment intensification in patients with PET-positive findings could have led to a similar BRFS between patients with a negative PET scan and patients with local or lymph node recurrence. Previously, Jilg et al. observed a high microscopic tumor spread in the surrounding lymphatic tissue of PSMA PET-positive nodes (6). Thus, contrary to the "Pokemet" approach (32) to metastatic PCa, we do not believe in stereotactic body radiotherapy of PET-positive nodes only but in eradicating microscopic spread to surrounding lymphatic pathways, dose escalation to macroscopic tumor burden, and at least concomitant use of ADT.

Nevertheless, $18 \%$ of the patients with PET-negative findings had a PSA of more than $0.2 \mathrm{ng} / \mathrm{mL}$ at the last follow-up. PSMA PET/CT may still underestimate the true extent of disease for detection of small-volume lymph nodes $(33,34)$. In our opinion, PSMA PET/CT should therefore not result in an omission of nomogram (35)-triggered radiotherapy volumes.

In our cohort, a significant correlation was present between a postradiotherapy PSA nadir of $0.1 \mathrm{ng} / \mathrm{mL}$ or less and a PSA of 0.2 $\mathrm{ng} / \mathrm{mL}$ or less at the last follow-up. This correlation mirrors recent data by Bartkowiak et al. (36) demonstrating that men with undetectable postradiotherapy PSA have lower rates of metastases and better overall survival. Previous data demonstrated a strong dependence of progression-free survival on presalvage radiotherapy PSA $(36,37)$. The analyses of Tendulkar et al. and Bartkowiak et al. $(36,37)$ in this regard incorporated patients of the pre-PSMA PET era, and the sRT approach was not based on advanced imaging allowing for treatment adaptation such as dose escalation to the macroscopic tumor recurrence and exclusion of metastatic disease. Most likely, this consideration explains why there was

TABLE 3

Outcome After Salvage Radiotherapy

\begin{tabular}{lccc}
\hline \multicolumn{1}{c}{ Parameter } & All patients $(n=90)$ & PET-positive $(n=42)$ & PET-negative $(n=48)$ \\
\hline Median follow-up $(\mathrm{mo})$ & $23(1-47)$ & & 42 \\
PSA at last follow-up $(n)$ & 90 & $0.07(<0.03-4.92)$ & $0.07(0.01-1.3)$ \\
Median PSA $(\mathrm{ng} / \mathrm{mL})$ & $0.07(<0.03-4.92)$ & $74 \%$ & $82 \%, P=0.377$ \\
PSA $\leq 0.1 \mathrm{ng} / \mathrm{mL}$ & $78 \%$ & $74 \%$ & $82 \%, P=0.662$ \\
PSA $\leq 0.2 \mathrm{ng} / \mathrm{mL}$ & $78 \%$ & 40 & 47 \\
PSA at last follow-up without ADT $(n)$ & 87 & $0.07(<0.03-4.92)$ & $0.07(<0.03-1.3)$ \\
Median PSA $(\mathrm{ng} / \mathrm{mL})$ & $0.07(<0.03-4.92)$ & $78 \%$ & $82 \%, P=0.191$ \\
PSA $\leq 0.1 \mathrm{ng} / \mathrm{mL}$ & $80 \%$ & $78 \%$ & $82 \%, P=0.392$ \\
PSA $\leq 0.2 \mathrm{ng} / \mathrm{mL}$ & $80 \%$ & & \\
Clinical progress & & & \\
Distant metastases & None & & \\
Death & None & &
\end{tabular}

Data in parentheses are ranges. 
TABLE 4

Comparison Between PSMA PET-Guided sRT and Non-Image-Guided sRT Studies

\begin{tabular}{|c|c|c|c|c|c|c|c|c|}
\hline \multirow[b]{2}{*}{ Study } & \multirow[b]{2}{*}{ Patients $(n)$} & \multicolumn{2}{|c|}{$\begin{array}{l}\text { sRT dose to } \\
\text { fossa (Gy) }\end{array}$} & \multirow{2}{*}{$\begin{array}{l}\text { sRT to pelvic } \\
\text { lymph nodes }\end{array}$} & \multirow{2}{*}{$\begin{array}{l}\text { Median PSA before } \\
\text { sRT (ng/mL) }\end{array}$} & \multirow{2}{*}{$\begin{array}{c}\text { Patients } \\
\text { with ADT (\%) }\end{array}$} & \multirow{2}{*}{$\begin{array}{c}\text { Guided by } \\
\text { PSMA PET/CT }\end{array}$} & \multirow{2}{*}{$\begin{array}{l}\text { BRFS } \\
\text { at } 2 y\end{array}$} \\
\hline & & Median & Range & & & & & \\
\hline Stephenson et al. (26) & 501 & 64.8 & $<59.4->70$ & $\begin{array}{l}\text { Yes (5\% } \\
\text { of patients) }\end{array}$ & 0.72 & 17 & No & $<60 \%$ \\
\hline Ervandian et al. (13) & 259 & 69 & $66-74$ & No & NA & 40.3 & No & $<70 \%$ \\
\hline Goenka et al. (12) & 285 & NA & $<70-\geq 70$ & $\begin{array}{l}\text { Yes ( } 7 \% \\
\text { of patients) }\end{array}$ & 0.4 & 31 & No & $<65 \%$ \\
\hline Stish et al. (27) & 1,106 & 68 & $64.8-70.2$ & $\begin{array}{l}\text { Yes ( } 4 \% \\
\quad \text { of patients) }\end{array}$ & 0.6 & 16.3 & No & $<70 \%$ \\
\hline Miyake et al. (28) & 61 & 70 & & Yes (NA) & 0.56 & 35.1 & No & $55 \%$ \\
\hline Abugharib et al. (29) & 657 & 68 & $59.4-72$ & Yes (NA) & 0.4 & 24 & No & $<70 \%$ \\
\hline Shipley et al. (2) & 376 & 64.8 & & No & 0.6 & $0^{*}$ & No & $80 \%$ \\
\hline Our study & 90 & $66^{\dagger}$ & $59.4-70.2$ & $\begin{array}{l}\text { Yes ( } 20 \% \\
\quad \text { of patients) }\end{array}$ & 0.44 & 27.8 & Yes & $78 \%$ \\
\hline
\end{tabular}

*Study arm without ADT.

†70 Gy (range, 67.2-72 Gy) to local recurrences.

$\mathrm{NA}=$ not available.

Shipley et al. (PSA nadir after sRT $+0.3 \mathrm{ng} / \mathrm{mL}$ ) and Stephenson et al. (PSA nadir after sRT $+0.1 \mathrm{ng} / \mathrm{mL}$ or continuous PSA increase) defined BRFS differently from us.

no such dependence of BRFS on the presalvage radiotherapy PSA in the present cohort: patients with a higher presalvage radiotherapy PSA - and thus, in most cases, with evidence of locoregional pelvic recurrence-received an individualized, escalated sRT based on the PSMA PET/CT results.

Our study had some limitations due mainly to its retrospective character: the treatment protocols and the follow-up procedure were not identical for all patients. Another issue was the radiation dose applied to PET-positive lesions in the prostatic fossa and to PETpositive pelvic lymph nodes: Zilli et al. (11) delivered 74 Gy to MRI-positive local relapses and D'Angelillo et al. (38) delivered up to 80 Gy to choline PET-positive lesions in the prostatic fossa. In comparison, dose escalation to macroscopic local recurrences in our study was performed with an overall lower dose of up to $72 \mathrm{~Gy}$. Thus, future studies should aim to assess the optimal sRT dose for PET-positive regions and clarify whether higher radiotherapy doses to PET-positive regions may enable deferral of ADT. Further shortcomings of the present analysis that limit final conclusions were the relatively short follow-up due to the only recent availability of PSMA PET/CT, as well as the differing duration of ADT in 26 of 90 patients $(29 \%)$ but with most patients $(88 \%)$ discontinuing ADT after $5 \mathrm{mo}$ and relatively long before the last PSA measurement. Hence, at best, our primary endpoint, BRFS, may serve as a surrogate marker for PCa-specific survival or overall survival.

\section{CONCLUSION}

We observed a 78\% BRFS rate after PSMA PET/CT-guided salvage radiotherapy and no differences in BRFS between patients with PET-positive and PET-negative lesions, indicating effective targeting of PET-positive lesions. To confirm the observed outcome improvement by PSMA PET/CT guidance, a prospective observational study at both institutions is being planned.

\section{DISCLOSURE}

No potential conflict of interest relevant to this article was reported.

\section{REFERENCES}

1. Han M, Partin AW, Zahurak M, Piantadosi S, Epstein JI, Walsh PC. Biochemical (prostate specific antigen) recurrence probability following radical prostatectomy for clinically localized prostate cancer. J Urol. 2003;169:517-523.

2. Shipley WU, Seiferheld W, Lukka HR, et al. Radiation with or without antiandrogen therapy in recurrent prostate cancer. N Engl J Med. 2017;376:417-428.

3. King CR. The dose-response of salvage radiotherapy following radical prostatectomy: a systematic review and meta-analysis. Radiother Oncol. 2016;121: 199-203.

4. Ghadjar P, Hayoz S, Bernhard J, et al. Acute toxicity and quality of life after dose-intensified salvage radiation therapy for biochemically recurrent prostate cancer after prostatectomy: first results of the randomized trial SAKK 09/10. J Clin Oncol. 2015;33:4158-4166.

5. Eiber M, Maurer T, Souvatzoglou M, et al. Evaluation of hybrid ${ }^{68} \mathrm{Ga}-\mathrm{PSMA}$ ligand PET/CT in 248 patients with biochemical recurrence after radical prostatectomy. J Nucl Med. 2015;56:668-674.

6. Jilg CA, Drendel V, Rischke HC, et al. Diagnostic accuracy of Ga-68-HBEDCC-PSMA-ligand-PET/CT before salvage lymph node dissection for recurrent prostate cancer. Theranostics. 2017;7:1770-1780.

7. Calais J, Czernin J, Cao M, et al. ${ }^{68} \mathrm{Ga}$-PSMA-11 PET/CT mapping of prostate cancer biochemical recurrence after radical prostatectomy in 270 patients with a PSA level of less than $1.0 \mathrm{ng} / \mathrm{mL}$ : impact on salvage radiotherapy planning. J Nucl Med. 2018;59:230-237.

8. Roach PJ, Francis R, Emmett L, et al. The impact of ${ }^{68}$ Ga-PSMA PET/CT on management intent in prostate cancer: results of an Australian prospective multicenter study. J Nucl Med. 2018;59:82-88.

9. Hope TA, Aggarwal R, Chee B, et al. Impact of ${ }^{68} \mathrm{Ga}$-PSMA-11 PET on management in patients with biochemically recurrent prostate cancer. $J$ Nucl Med. 2017;58:1956-1961.

10. Calais J, Fendler WP, Eiber M, et al. Actual impact of ${ }^{68}$ Ga-PSMA-11 PET/CT on the management of prostate cancer patients with biochemical recurrence. J Nucl Med. 2018;59:434-441. 
11. Zilli T, Jorcano S, Peguret N, et al. Results of dose-adapted salvage radiotherapy after radical prostatectomy based on an endorectal MRI target definition model. Am J Clin Oncol. 2017;40:194-199.

12. Goenka A, Magsanoc JM, Pei X, et al. Long-term outcomes after high-dose postprostatectomy salvage radiation treatment. Int J Radiat Oncol Biol Phys. 2012; 84:112-118.

13. Ervandian M, Høyer M, Petersen SE, et al. Salvage radiation therapy following radical prostatectomy: a national Danish study. Acta Oncol. 2016;55: 598-603.

14. Zschaeck S, Wust P, Beck M, et al. Intermediate-term outcome after PSMA-PET guided high-dose radiotherapy of recurrent high-risk prostate cancer patients. Radiat Oncol. 2017;12:140.

15. Emmett L, Van Leeuwen $P$, Nandurkar R, et al. Treatment outcomes from ${ }^{68}$ GaPSMA PET CT informed salvage radiation treatment in men with rising PSA following radical prostatectomy: prognostic value of a negative PSMA PET. J Nucl Med. 2017;58:1972-1976.

16. Wiegel T, Bartkowiak D, Bottke D, et al. Prostate-specific antigen persistence after radical prostatectomy as a predictive factor of clinical relapse-free survival and overall survival: 10-year data of the ARO 96-02 trial. Int J Radiat Oncol Biol Phys. 2015;91:288-294.

17. Bianchi L, Nini A, Bianchi M, et al. The role of prostate-specific antigen persistence after radical prostatectomy for the prediction of clinical progression and cancer-specific mortality in node-positive prostate cancer patients. Eur Urol. 2016;69:1142-1148.

18. General Assembly of the World Medical Association. World Medical Association Declaration of Helsinki: ethical principles for medical research involving human subjects. J Am Coll Dent. 2014;81:14-18.

19. Weineisen M, Simecek J, Schottelius M, Schwaiger M, Wester H-J. Synthesis and preclinical evaluation of DOTAGA-conjugated PSMA ligands for functional imaging and endoradiotherapy of prostate cancer. EJNMMI Res. 2014;4:63.

20. Zamboglou C, Wieser G, Hennies S, et al. MRI versus ${ }^{68}$ Ga-PSMA PET/CT for gross tumour volume delineation in radiation treatment planning of primary prostate cancer. Eur J Nucl Med Mol Imaging. 2016;43:889-897.

21. Fendler WP, Eiber M, Beheshti M, et al. ${ }^{68} \mathrm{Ga}$-PSMA PET/CT: joint EANM and SNMMI procedure guideline for prostate cancer imaging: version 1.0. Eur J Nucl Med Mol Imaging. 2017;44:1014-1024.

22. Prostate post-op. RTOG Foundation website. https://www.rtog.org/CoreLab/ ContouringAtlases/ProstatePostOp.aspx. Accessed October 4, 2018.

23. Lawton CAF, Michalski J, El-Naqa I, et al. RTOG GU radiation oncology specialists reach consensus on pelvic lymph node volumes for high-risk prostate cancer. Int J Radiat Oncol Biol Phys. 2009;74:383-387.

24. Albisinni S, Artigas C, Aoun F, et al. Clinical impact of ${ }^{68} \mathrm{Ga}$-prostate-specific membrane antigen (PSMA) positron emission tomography/computed tomography
(PET/CT) in patients with prostate cancer with rising prostate-specific antigen after treatment with curative intent: preliminary analysis of a multidisciplinary approach. BJU Int. 2017;120:197-203.

25. Bluemel C, Linke F, Herrmann $\mathrm{K}$, et al. Impact of ${ }^{68} \mathrm{Ga}$-PSMA PET/CT on salvage radiotherapy planning in patients with prostate cancer and persisting PSA values or biochemical relapse after prostatectomy. EJNMMI Res. 2016;6:78.

26. Stephenson AJ, Shariat SF, Zelefsky MJ, et al. Salvage radiotherapy for recurrent prostate cancer after radical prostatectomy. JAMA. 2004;291:1325-1332.

27. Stish BJ, Pisansky TM, Harmsen WS, et al. Improved metastasis-free and survival outcomes with early salvage radiotherapy in men with detectable prostate-specific antigen after prostatectomy for prostate cancer. J Clin Oncol. 2016;34:3864-3871.

28. Miyake M, Tanaka N, Asakawa I, et al. Proposed salvage treatment strategy for biochemical failure after radical prostatectomy in patients with prostate cancer: a retrospective study. Radiat Oncol. 2014;9:208.

29. Abugharib A, Jackson WC, Tumati V, et al. Very early salvage radiotherapy improves distant metastasis-free survival. J Urol. 2017;197:662-668.

30. Thompson IM, Valicenti RK, Albertsen P, et al. Adjuvant and salvage radiotherapy after prostatectomy: AUA/ASTRO guideline. J Urol. 2013;190:441-449.

31. Henkenberens C, Von Klot CA, Ross TL, et al. ${ }^{68}$ Ga-PSMA ligand PET/CT-based radiotherapy for lymph node relapse of prostate cancer after primary therapy delays initiation of systemic therapy. Anticancer Res. 2017;37:1273-1279.

32. Murphy DG, Sweeney CJ, Tombal B. "Gotta Catch 'em All”, or Do We? Pokemet Approach to Metastatic Prostate Cancer. Eur Urol. 2017;72:1-3.

33. Maurer T, Gschwend JE, Rauscher I, et al. Diagnostic efficacy of ${ }^{68}$ GalliumPSMA positron emission tomography compared to conventional imaging for lymph node staging of 130 consecutive patients with intermediate to high risk prostate cancer. J Urol. 2016;195:1436-1443.

34. van Leeuwen PJ, Emmett L, Ho B, et al. Prospective evaluation of ${ }^{68}$ galliumprostate-specific membrane antigen positron emission tomography/computed tomography for preoperative lymph node staging in prostate cancer. BJU Int. 2017; 119:209-215.

35. Wu R, Woodford H, Capp A, et al. A prospective study of nomogram-based adaptation of prostate radiotherapy target volumes. Radiat Oncol. 2015;10:243.

36. Bartkowiak D, Thamm R, Bottke D, et al. Prostate-specific antigen after salvage radiotherapy for postprostatectomy biochemical recurrence predicts long-term outcome including overall survival. Acta Oncol. 2018;57:362-367.

37. Tendulkar RD, Agrawal S, Gao T, et al. Contemporary update of a multi-institutional predictive nomogram for salvage radiotherapy after radical prostatectomy. J Clin Oncol. 2016;34:3648-3654.

38. D'Angelillo RM, Sciuto R, Ramella $\mathrm{S}$, et al. ${ }^{18} \mathrm{~F}$-choline positron emission tomography/computed tomography-driven high-dose salvage radiation therapy in patients with biochemical progression after radical prostatectomy: feasibility study in 60 patients. Int J Radiat Oncol Biol Phys. 2014;90:296-302. 